\title{
BMJ Open Association between fluid intake and kidney function, and survival outcomes analysis: a nationwide population-based study
}

\author{
Li-Wei Wu, ${ }^{1,2,3}$ Wei-Liang Chen, ${ }^{1,2,3}$ Fang-Yih Liaw, ${ }^{1}$ Yu-Shan Sun, ${ }^{1}$ \\ Hui-Fang Yang, ${ }^{1}$ Chung-Ching Wang, ${ }^{1}$ Chien-Ming Lin, ${ }^{3}$ Yu-Tzu Tsao ${ }^{4,5}$
}

To cite: Wu L-W, Chen W-L, Liaw F-Y, et al. Association between fluid intake and kidney function, and survival outcomes analysis: a nationwide population-based study. BMJ Open 2016;6: e010708. doi:10.1136/ bmjopen-2015-010708

- Prepublication history for this paper is available online. To view these files please visit the journal online (http://dx.doi.org/10.1136/ bmjopen-2015-010708).

Received 1 December 2015 Revised 16 March 2016 Accepted 28 April 2016
CrossMark

For numbered affiliations see end of article.

Correspondence to Dr Yu-Tzu Tsao; tsaoyutzu@gmail.com

\section{ABSTRACT}

Objectives: Fluid intake, one of the most common daily activities, has not been well studied in chronic kidney disease (CKD) populations, and clinical outcomes are rarely addressed. The aim of this nationwide study is to explore the influence of daily fluid intake on cardiovascular and all-cause mortality and its association with renal function.

Design: Observational cohort study.

Participants: In all, 2182 participants aged more than 20 years participated in the Third National Health and Nutrition Examination Survey (1988-1994).

Main outcome measures: Survival outcomes in patients with or without CKD, using multiple variable adjusted Cox proportional hazard models.

Results: In a longitudinal survey with a median follow-up length of 15.4 years, 1080 participants died and 473 cardiovascular deaths were recorded. For allcause mortality in the CKD group, individuals in the highest quartile of fluid intake ( $\geqq 3.576 \mathrm{~L} /$ day) had better survival outcomes than those in the lowest quartile of fluid intake ( $\leq 2.147 \mathrm{~L} /$ day) $(p=0.029)$ after adjustment of several pertinent variables.

Conclusions: Although the interpretation of this observational study was limited by the failure to identify the compositions of ingested fluids, adequate hydration may offer some advantages in patients with CKD. However, the underlying pathophysiological mechanisms of the responses of normal and injured kidneys to chronic changes in fluid consumption warrant further investigation.

\section{INTRODUCTION}

Chronic kidney disease (CKD) has become a global public health problem that is strongly associated with cardiovascular disease, endstage renal disease and mortality. ${ }^{1}{ }^{2}$ Although a variety of factors contributing to renal progression and survival outcomes have been elucidated in patients with CKD, the association between fluid intake and mortality has not been established. It is widely recognised that adequate hydration is

\section{Strengths and limitations of this study}

- The study used a nationwide population-based data set.

- The study explored the influence of daily fluid intake, and cardiovascular and all-cause mortality, and its association with renal function.

- The Dietary Food Frequency Questionnaire in the National Health and Nutrition Examination Survey III survey may not provide information on longterm diets nor accurately reflect actual intake.

essential for the body to maintain normal physiological function, including circulation, nutrient transport, excretion and regulation of body temperature. The European Food Safety Agency (EFSA) recommends a daily total water intake of $2.5 \mathrm{~L}$ for men and $2.0 \mathrm{~L}$ for women. ${ }^{3}$ However, there is no evidencebased recommendation regarding fluid intake in CKD. There is accumulating evidence from animal and human studies regarding the beneficial effects of water intake on the kidney. ${ }^{4-9}$ In animal models, increased water intake has been associated with decreased proteinuria and delayed progression of $\mathrm{CKD}^{7}{ }^{8}$ Information from human observational studies indicates a positive association between increased water intake and renal function. ${ }^{4} 5$ Chronic mild-to-moderate dehydration has been associated with several disease states, such as fatal chronic heart disease and cardiovascular disease (CVD).${ }^{10}$ Although low fluid intake appears to correlate with increased comorbidities and reduced renal function, there is little information concerning the relationship between daily fluid intake and mortality in patients with CKD. Therefore, the purpose of our study was to determine whether low daily fluid intake was an independent risk factor for survival in CKD. 


\section{MATERIALS AND METHODS}

\section{Study design and participants}

The third National Health and Nutrition Examination Survey (NHANES III), a cross-sectional survey of a representative sample of the US population from 1988 to 1994, obtained a random sample of non-institutionalised US citizens, using a stratified, multistage and cluster sampling design. Trained examiners obtained pertinent information during a home interview, including age, gender, race and medical history. In addition, dietary interviews were administered to all examinees by a trained dietary interviewer in a mobile examination centre. Nutrient intake was determined based on foods and beverages reported via $24 \mathrm{~h}$ dietary recall. Questionnaire data on food intake, intake of plain drinking water and salt use were also obtained. The reliability and validity of the Dietary Food Frequency Questionnaire for dietary use has been assessed previously. ${ }^{11}{ }^{12}$ Vital signs, anthropometric, physiological and laboratory investigations were included in this survey. Detailed descriptions of NHANES methodology and data collection have been published. ${ }^{13}$ The NHANES III study received NCHS Institutional Review Board approval, and informed consent was acquired from participants prior to the start of the study.

\section{Follow-up data}

The NHANES III was not only a cross-sectional study but also obtained mortality follow-up data from the time of study participation. Mortality follow-up data (NHANES III Linked Mortality File) were provided by the National Center for Health Statistics according to a probabilistic match between NHANES III participants and National Death Index death certificate records. Follow-up data were obtained from the time of NHANES III study participation through 31 December 2006. ${ }^{14}$

\section{Participant exclusion criteria}

Among these populations, eligible individuals with incomplete data regarding daily fluid intake, renal function measurements, laboratory and clinical examinations or household interview, were excluded. Moreover, in order to minimise confounding, we excluded participants who were pregnant, used lithium or diuretics or who suffered from congestive heart failure.

\section{Measurement of daily fluid intake}

Daily total fluid intake was assessed using the $24 \mathrm{~h}$ dietary recall questionnaire. The questionnaire used standardised questions to help the respondents recall and describe the foods and beverages that they consumed. Daily total fluid intake was estimated from all foods and beverages consumed during the previous $24 \mathrm{~h}$, including plain drinking water, spring water, and water contained in foods and other beverages. Owing to the absence of data regarding daily recommended fluid intake, we categorised daily fluid intake into four groups. The quartiles of daily fluid intake were as follows: Q1 $\leqq 2.147 \mathrm{~L} /$ day, $2.147 \mathrm{~L} /$ day $<\mathrm{Q} 2$ $\leqq 2.789 \mathrm{~L} /$ day, $2.789 \mathrm{~L} /$ day $<\mathrm{Q} 3 \leqq 3.576 \mathrm{~L} /$ day and $\mathrm{Q} 4$ $\geq 3.576 \mathrm{~L} /$ day.

\section{Measurement of renal function}

Renal function was evaluated based on serum creatinine values, and calculation of the estimated glomerular filtration rate (eGFR) was based on the abbreviated Modification of Diet in Renal Disease study formula: eGFR $=186.3 \times(\text { serum creatinine in } \mathrm{mg} / \mathrm{dL})^{-1.154} \times$ age ${ }^{-0.203} \times(0.742$ if female $) \times(1.21$ if black $)$. The presence of CKD was defined as either an eGFR of $\leqq 60 \mathrm{~mL} / \mathrm{min} /$ $1.73 \mathrm{~m}^{2}$ or the presence of albuminuria. The criterion for albuminuria was spot urinary albumin-to-creatinine ratio $\geqq 30 \mathrm{mg} / \mathrm{g}$.

\section{Covariates}

Age, gender, race and smoking status were obtained by self-reporting. Body mass index (BMI) was calculated as weight in kilograms divided by the square of height in metres. A mercury sphygmomanometer was used by an NHANES physician to obtain three to four blood pressure measurements. Averaged systolic and diastolic blood pressure readings were obtained. Diabetes mellitus (DM) was defined by self-reporting of a physician's diagnosis, a fasting glucose $\geqq 126 \mathrm{mg} / \mathrm{dL}$ or the use of diabetes medications (including insulin injections or oral hypoglycaemic agents). Serum uric acid levels were measured using a Hitachi 737 automated multichannel chemistry analyser (Boehringer Mannheim Diagnostics, Indianapolis, Indiana, USA). Chemical analyses of total cholesterol and triglycerides were performed by the Lipoprotein Analytical Laboratory at Johns Hopkins University, Baltimore, Maryland. Apolipoprotein AI were measured by radial immunodiffusion or by rate immunonephelometric assay. All measurements were completed using standardised methods with documented accuracy, with respect to the Centres for Disease Control and Prevention (CDC) reference methods. Co-morbidities including cancer and chronic bronchitis were ascertained by self-reporting. ${ }^{15}$ The HOMA-IR score was calculated using the HOMA-IR formula (HOMA-IR=fasting insulin $(\mathrm{mIU} / \mathrm{L}) \times$ fasting glucose $(\mathrm{mmol} / \mathrm{L}) / 22.5 ;$ fasting glucose used in this equation was measured by the hexokinase method and fasting insulin was measured using Merocodia Insulin ELISA). Details concerning data quality control have been published elsewhere. ${ }^{16}$

\section{Statistical analysis}

All statistical analyses were computed using SPSS (V.18.0 for Windows, SPSS, Inc, Chicago, Illinois, USA) complex samples to incorporate sample weights, and to adjust for the clusters and strata of the complex sample design. Descriptive results are expressed as means \pm SDs for continuous variables, and as numbers and percentages for categorical variables. Differences in the characteristics of the participants were examined by the $\chi^{2}$ test, Student t test or Mann-Whitney U-test, as appropriate. We used quintile-based analysis by dividing the daily total fluid 
intake into quartiles with the subjects in the lowest quartiles serving as the reference group. For the longitudinal follow-up, overall survival was determined using the Kaplan-Meier method, based on the quintiles of daily total fluid intake. Univariable and multivariable adjusted Cox proportional hazard models were performed to investigate the association between all-cause mortality and daily total fluid intake after controlling for age, gender, race-ethnicity, BMI, waist, white blood cell count, eGFR, high-density lipoprotein (HDL) cholesterol, apolipoprotein AI, serum glucose, plasma fibrinogen, albumin, serum $\mathrm{C}$ reactive protein, smoke, cancer, emphysema, DM and arthritis. Univariable and multivariable adjusted Cox proportional hazard models were performed to investigate the association between the cardiovascular mortality and daily total fluid intake after controlling for age, gender, race-ethnicity, BMI, waist, white blood cell count, eGFR, HDL cholesterol, apolipoprotein $\mathrm{AI}$, serum glucose, plasma fibrinogen, albumin, serum $\mathrm{C}$ reactive protein, smoke, cancer, emphysema, $\mathrm{DM}$ and arthritis.

\section{RESULTS}

The study included a total of 2182 participants aged more than 20 years who completed the dietary questionnaire and laboratory examinations. The characteristics of the participants by quartiles of daily total fluid intake were summarised in table 1 . Of the 2182 participants, the mean age was 66.6 years with a SD of 10.24 years. Participants in a higher quartile of daily total fluid intake were inclined to have a higher BMI, waist circumference, eGFR, haemoglobin, serum low-density lipoprotein cholesterol and serum glucose. Participants with higher daily total fluid intake tended to have a history of smoking.

Table 2 presents the descriptive data for the CKD $(\mathrm{N}=933)$ and non-CKD groups $(\mathrm{N}=1249)$. The mean ages for the CKD and non-CKD groups were 70.39 years and 63.76 years, respectively. The CKD group had a lower daily total fluid intake than the non-CKD group (2865.48 mL/day vs $3049 \mathrm{~mL} /$ day, $\mathrm{p}<0.001)$. There were significant differences in systolic blood pressure $(\mathrm{p}<0.001)$, eGFR $(\mathrm{p}<0.001)$, haemoglobin $(\mathrm{p}<0.001)$, serum cholesterol $(\mathrm{p}<0.001)$, plasma fibrinogen $(\mathrm{p}<0.001)$, serum potassium $(\mathrm{p}<0.001)$, serum phosphorus $(p=0.037)$, serum blood urea nitrogen $(\mathrm{p}<0.001)$, serum creatinine $(\mathrm{p}<0.001)$ and serum albumin $(p<0.001)$ between the two groups. The CKD group had a higher prevalence of cancer history than the non-CKD group.

In this study, the median length of follow-up was 15.4 years. Overall, there were 1080 all-cause deaths and 473 cardiovascular deaths. In the non-CKD group, there were 137 deaths (48 cardiovascular deaths) among participants who consumed $\leqq 2.147 \mathrm{~L} /$ day, 137 deaths (56 cardiovascular deaths) among participants who consumed 2.147-2.789 L/day, 131 deaths (53 cardiovascular deaths) among participants who consumed 2.789$3.576 \mathrm{~L} /$ day and 126 deaths (48 cardiovascular deaths) among participants who consumed $\geq 3.576 \mathrm{~L} /$ day. Concerning the survival curve of the non-CKD group (figure 1), the probability of survival was highest in the $\geq 3.576 \mathrm{~L} /$ day category and lowest in the $\leqq 2.147 \mathrm{~L} /$ day category $(\mathrm{p}=0.001)$.

In the CKD group, there were 161 deaths (85 cardiovascular deaths) among participants who consumed $\leqq 2.147 \mathrm{~L} /$ day, 145 deaths (63 cardiovascular deaths) among participants who consumed 2.147-2.789 L/day, 142 deaths (71 cardiovascular deaths) among participants who consumed 2.789-3.576 L/day and 101 deaths (49 cardiovascular deaths) among participants who consumed $\geq 3.576 \mathrm{~L} /$ day. Concerning the survival curve of the CKD group (figure 2), the probability of survival was highest in the $\geq 3.576 \mathrm{~L} /$ day category and lowest in the $\leqq 2.147 \mathrm{~L} /$ day category $(\mathrm{p}=0.009)$.

Regarding the all-cause mortality in the CKD group (table 3), the unadjusted HRs for each quartile of increasing fluid intake were $0.944 \quad(\mathrm{p}=0.613), 0.939$ $(\mathrm{p}=0.586)$ and $0.720 \quad(\mathrm{p}=0.010)$ for fluid intakes of 2.147-2.789 L/day, 2.789-3.576 L/day and $\geq 3.576 \mathrm{~L} /$ day, respectively, compared with $\leqq 2.147 \mathrm{~L} /$ day. The multivariable adjusted HRs for each quartile of increasing fluid intake were $0.933(\mathrm{p}=0.562), 0.914(\mathrm{p}=0.481)$ and 0.741 ( $\mathrm{p}=0.029$ ) for fluid intake of $2.147-2.789 \mathrm{~L} /$ day, 2.789 $3.576 \mathrm{~L} /$ day and $\geq 3.576 \mathrm{~L} /$ day, respectively, compared with $\leqq 2.147 \mathrm{~L} /$ day.

Regarding cardiovascular mortality in the CKD group (table 4), the unadjusted HRs for each quartile of increasing fluid intake were $0.853(\mathrm{p}=0.141), 0.844$ $(\mathrm{p}=0.145)$ and $0.841 \quad(\mathrm{p}=0.173)$ for fluid intakes of 2.147-2.789 L/day, $2.789-3.576 \mathrm{~L} /$ day and $\geq 3.576 \mathrm{~L} /$ day, respectively, compared with $\leqq 2.147 \mathrm{~L} /$ day. The multivariable adjusted HRs for each quartile of increasing fluid intake were $0.847(\mathrm{p}=0.179), 0.870(\mathrm{p}=0.198)$ and 0.827 $(\mathrm{p}=0.236)$ for fluid intake of $2.147-2.789 \mathrm{~L} /$ day, 2.789 $3.576 \mathrm{~L} /$ day and $\geq 3.576 \mathrm{~L} /$ day, respectively, compared with $\leqq 2.147 \mathrm{~L} /$ day. Compared with the lowest quartile of fluid intake in the CKD group, a significant association was observed between the highest quartile of fluid intake and all-cause mortality in the univariable and multivariable adjusted analyses but not in cardiovascular mortality.

\section{DISCUSSION}

Our study is a longitudinal survey of noninstitutionalised US citizens that correlates all-cause mortality in non-CKD and CKD populations with total fluid intake and also cardiovascular outcomes. Our study showed that there was no benefit in cardiovascular mortality in patients with CKD with higher daily fluid intake as already demonstrated by the cross-sectional NHANES data from 2005 to 2006 by Sontrop et al. ${ }^{17}$ The paper by Sontrop showed the total water intake as opposed to total fluid intake had no significant association with 
Table 1 Characteristics of study participants

\begin{tabular}{|c|c|c|c|c|c|c|}
\hline \multirow[b]{2}{*}{ Characteristic } & \multicolumn{6}{|c|}{ Quartiles of fluid intake (L/day) } \\
\hline & $\begin{array}{l}\text { Q1 (<2.147) } \\
\mathrm{N}=545\end{array}$ & $\begin{array}{l}Q 2(2.147 \text { to } 2.789) \\
N=546\end{array}$ & $\begin{array}{l}\text { Q3 (2.789 to } 3.576) \\
\mathrm{N}=546\end{array}$ & $\begin{array}{l}Q 4(\geq 3.576) \\
N=545\end{array}$ & $\begin{array}{l}\text { Total } \\
\mathrm{N}=2182\end{array}$ & p Value \\
\hline \multicolumn{7}{|l|}{ Continuous variables } \\
\hline Age, mean (SD) & $68.81(10.50)$ & $67.45(10.36)$ & $66.15(9.97)$ & $63.98(9.51)$ & $66.60(10.24)$ & $<0.001$ \\
\hline BMI, mean (SD) & $26.36(4.829)$ & $27.00(4.800)$ & $27.41(5.237)$ & $27.82(5.341)$ & $27.15(5.083)$ & $<0.001$ \\
\hline Waist circumference $(\mathrm{cm})$ & $94.28(12.19)$ & $96.91(11.88)$ & $98.05(12.70)$ & $99.68(13.13)$ & $97.23(12.63)$ & $<0.001$ \\
\hline Systolic blood pressure (mm HG) & 139.55 (22.53) & $138.45(22.86)$ & $136.60(22.12)$ & $136.03(20.89)$ & $137.66(22.14)$ & 0.037 \\
\hline eGFR & $60.82(13.53)$ & $62.22(13.80)$ & $62.83(12.55)$ & $64.18(13.41)$ & $62.51(13.38)$ & $<0.001$ \\
\hline WCC (1000 cells $/ \mathrm{mL})$ & $6.99(2.31)$ & $6.87(2.81)$ & $7.14(2.49)$ & $7.15(2.03)$ & $7.04(2.26)$ & 0.134 \\
\hline Haemoglobin $(\mathrm{g} / \mathrm{dL})$, mean $(\mathrm{SD})$ & $13.62(1.31)$ & $13.87(1.33)$ & $13.98(1.34)$ & $14.20(1.31)$ & $13.92(1.34)$ & $<0.001$ \\
\hline Serum cholesterol (mg/dL) & $229.28(47.48)$ & $223.58(43.34)$ & $222.36(43.19)$ & $223.64(41.36)$ & 224.71 (43.95) & 0.043 \\
\hline Serum HDL cholesterol (mg/dL) & $53.68(15.47)$ & $51.60(16.09)$ & $51.10(15.57)$ & $51.30(16.30)$ & $51.92(15.88)$ & 0.027 \\
\hline Serum triglycerides (mg/dL) & $154.64(96.54)$ & $157.68(103.98)$ & $160.93(113.19)$ & $161.95(112.64)$ & $158.80(106.78)$ & 0.666 \\
\hline Serum apolipoprotein $\mathrm{Al}(\mathrm{mg} / \mathrm{dL})$ & $152.33(26.56)$ & $146.84(26.43)$ & $146.69(26.06)$ & $146.64(26.81)$ & $148.12(26.56)$ & $<0.001$ \\
\hline Plasma fibrinogen (mg/dL) & $318.11(96.84)$ & $322.13(101.86)$ & $323.74(90.67)$ & 324.53 (95.34) & 322.14 (96.22) & 0.710 \\
\hline Serum $C$ reactive protein $(\mathrm{mg} / \mathrm{dL})$ & $0.51(0.71)$ & $0.50(0.88)$ & $0.46(0.60)$ & $0.51(0.75)$ & $0.49(0.74)$ & 0.671 \\
\hline Serum sodium (mmol/L) & $141.93(2.53)$ & $141.80(2.80)$ & $141.79(2.38)$ & $141.51(2.92)$ & $141.76(2.67)$ & 0.061 \\
\hline Serum potassium $(\mathrm{mmol} / \mathrm{L})$ & $4.03(0.32)$ & $4.06(0.35)$ & $4.04(0.36)$ & $4.05(0.36)$ & $4.04(0.35)$ & 0.694 \\
\hline Serum total calcium (mmol/L) & $9.34(0.44)$ & $9.37(0.45)$ & $9.36(0.42)$ & $9.36(0.44)$ & $9.36(0.44)$ & 0.863 \\
\hline Serum phosphorus (mmol/L) & $3.47(0.50)$ & $3.42(0.51)$ & $3.43(0.49)$ & $3.43(0.50)$ & $3.44(0.50)$ & 0.413 \\
\hline Serum glucose $(\mathrm{mg} / \mathrm{dL})$ & $101.84(35.44)$ & $105.17(39.34)$ & 102.42 (32.63) & $111.98(55.94)$ & $105.35(41.98)$ & $<0.001$ \\
\hline Serum blood urea nitrogen (mg/dL) & $17.36(6.14)$ & $17.08(5.84)$ & $16.96(6.44)$ & $16.59(6.66)$ & $17.00(6.28)$ & 0.238 \\
\hline Serum creatinine (mg/dL) & $1.14(0.55)$ & $1.14(0.28)$ & $1.15(0.35)$ & $1.15(0.31)$ & $1.14(0.39)$ & 0.964 \\
\hline Serum albumin (g/dL) & $4.14(0.34)$ & $4.16(0.32)$ & $4.17(0.32)$ & $4.17(0.35)$ & $4.16(0.33)$ & 0.214 \\
\hline Urinary albumin $(\mu \mathrm{g} / \mathrm{mL})$ & $43.34(271.38)$ & $31.73(133.15)$ & 40.95 (196.61) & $64.02(437.72)$ & 44.99 (283.29) & 0.299 \\
\hline HOMA-IR & $3.48(6.93)$ & $3.19(3.97)$ & $3.75(6.39)$ & $4.56(14.94)$ & $3.75(9.06)$ & 0.075 \\
\hline Urine albumin to creatinine ratio $(\mathrm{mg} / \mathrm{g})$, mean $(\mathrm{SD})$ & $0.57(4.27)$ & $0.33(2.07)$ & $0.39(1.55)$ & $0.60(3.83)$ & $0.47(3.14)$ & 0.430 \\
\hline \multicolumn{7}{|l|}{ Categorical variables } \\
\hline Male, $n(\%)$ & $196(36.0)$ & $271(49.6)$ & $300(54.9)$ & $330(60.6)$ & $1097(50.3)$ & 0.004 \\
\hline Non-Hispanic white, $\mathrm{n}(\%)$ & $310(56.9)$ & $331(60.6)$ & $346(63.4)$ & $360(66.1)$ & $1347(61.7)$ & 0.050 \\
\hline Ever smoker, n (\%) & $277(50.8)$ & $303(55.5)$ & $314(57.5)$ & $348(63.9)$ & $1242(56.9)$ & 0.001 \\
\hline Diabetes mellitus, $n(\%)$ & $57(10.5)$ & $67(12.3)$ & $64(11.7)$ & $68(12.5)$ & $256(11.7)$ & 0.638 \\
\hline Cancer, n (\%) & $81(14.9)$ & 96 (17.6) & $105(19.3)$ & $89(16.4)$ & $371(17.0)$ & 0.519 \\
\hline Emphysema, n (\%) & $24(4.4)$ & $13(2.4)$ & $27(4.9)$ & $20(3.7)$ & $84(3.8)$ & 0.201 \\
\hline Arthritis, n (\%) & $238(43.7)$ & 209 (38.3) & 205 (37.5) & 209 (38.3) & $861(39.5)$ & 0.207 \\
\hline
\end{tabular}

BMI, body mass index; eGFR, estimated glomerular filtration rate; HDL, high-density lopoprotein; WCC, white cell count. 
Table 2 Participants characteristics by the presence of CKD

\begin{tabular}{|c|c|c|c|}
\hline & $\begin{array}{l}\text { CKD group } \\
N=933\end{array}$ & $\begin{array}{l}\text { Non-CKD group } \\
\mathrm{N}=1249\end{array}$ & p Value \\
\hline Age, mean (SD) & $70.39(9.58)$ & $63.76(9.79)$ & $<0.001$ \\
\hline Fluid intake (mL/day), mean (SD) & $2865.48(1178.54)$ & 3049.16 (1215.68) & $<0.001$ \\
\hline BMI, mean (SD) & $27.24(5.15)$ & $27.08(5.03)$ & 0.475 \\
\hline Waist circumference $(\mathrm{cm})$ & $97.43(12.57)$ & $97.07(12.68)$ & 0.510 \\
\hline Systolic blood pressure (mm HG) & $140.61(23.58)$ & 135.40 (20.69) & $<0.001$ \\
\hline eGFR & $50.68(8.17)$ & $71.35(8.95)$ & $<0.001$ \\
\hline Haemoglobin (g/dL), mean (SD) & $13.66(1.34)$ & $14.11(1.31)$ & $<0.001$ \\
\hline Serum cholesterol (mg/dL) & $228.88(45.87)$ & $221.60(42.21)$ & $<0.001$ \\
\hline Serum triglycerides (mg/dL) & $162.23(102.42)$ & $156.23(109.89)$ & 0.195 \\
\hline Serum HDL cholesterol (mg/dL) & $52.14(15.68)$ & $51.75(16.04)$ & 0.578 \\
\hline Serum apolipoprotein $\mathrm{Al}$ (mg/dL) & $149.31(26.90)$ & $147.24(26.28)$ & 0.072 \\
\hline Plasma fibrinogen (mg/dL) & $334.22(98.61)$ & 313.27 (93.47) & $<0.001$ \\
\hline Serum $\mathrm{C}$ reactive protein $(\mathrm{mg} / \mathrm{dL})$ & $0.53(0.79)$ & $0.47(0.70)$ & 0.087 \\
\hline Serum sodium $(\mathrm{mmol} / \mathrm{L})$ & $141.80(2.77)$ & $141.73(2.59)$ & 0.551 \\
\hline Serum potassium (mmol/L) & $4.09(0.37)$ & $4.01(0.33)$ & $<0.001$ \\
\hline Serum total calcium (mmol/L) & $9.37(0.46)$ & $9.34(0.43)$ & 0.131 \\
\hline Serum phosphorus (mmol/L) & $3.46(0.49)$ & $3.42(0.51)$ & 0.037 \\
\hline Serum glucose $(\mathrm{mg} / \mathrm{dL})$ & $106.41(43.21)$ & $104.56(41.04)$ & 0.308 \\
\hline Serum blood urea nitrogen $(\mathrm{mg} / \mathrm{dL})$ & $19.40(7.35)$ & $15.20(4.58)$ & $<0.001$ \\
\hline Serum creatinine (mg/dL) & $1.32(0.51)$ & $1.01(0.15)$ & $<0.001$ \\
\hline Serum albumin $(\mathrm{g} / \mathrm{dL})$ & $4.13(0.32)$ & $4.19(0.34)$ & $<0.001$ \\
\hline Male, $n(\%)$ & $374(40.1)$ & 723 (57.9) & $<0.001$ \\
\hline Non-Hispanic white, n (\%) & $622(66.7)$ & $725(58.0)$ & $<0.001$ \\
\hline Ever smoker, n (\%) & $474(50.8)$ & $768(61.5)$ & $<0.001$ \\
\hline Diabetes mellitus, $\mathrm{n}(\%)$ & $139(11.1)$ & $117(12.5)$ & 0.358 \\
\hline Cancer, n (\%) & 195 (20.9) & $176(14.1)$ & 0.048 \\
\hline Emphysema, n (\%) & $39(4.2)$ & $45(3.6)$ & 0.543 \\
\hline Arthritis, $\mathrm{n}(\%)$ & $445(35.6)$ & $416(44.6)$ & $<0.001$ \\
\hline
\end{tabular}

BMI, body mass index; CKD, chronic kidney disease; eGFR, estimated glomerular filtration rate; HDL, high-density lipoprotein.

cardiovascular disease, but was significantly correlated with a positive outcome in the reduced incidence of chronic kidney disease. When total water intake was categorised into the intake of plain water and other beverages, CKD was associated with low intake of plain water (adjusted ORs 2.36, 95\% CI 1.10 to 5.06) but not the other beverages. ${ }^{17}$ However, the study was a cross-

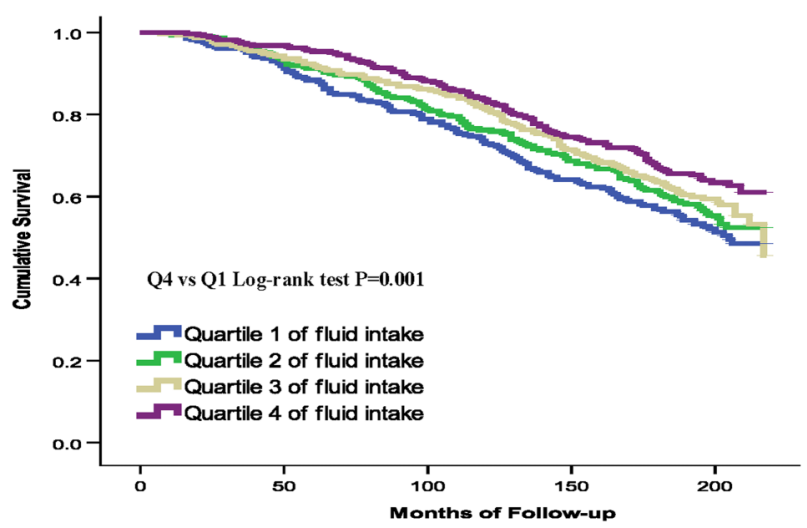

Figure 1 Kaplan-Meier plot of association of daily fluid intake quartiles with all-cause mortality in participants without chronic kidney disease. sectional observational analysis that has limitations for making causal inferences. Our is the only study that does not suffer the dietary and fluid intake recall bias issue and actually measures $24 \mathrm{~h}$ urine volume. It is a longitudinal study over 7 years and involved over 2000 participants. The Sontrop paper measured both dietary and fluid intake and showed the significant correlation

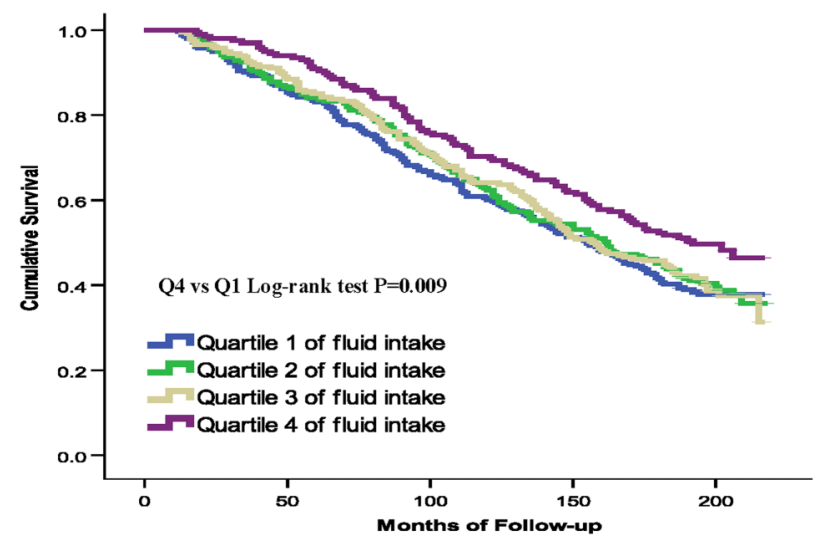

Figure 2 Kaplan-Meier plot of association of daily fluid intake quartiles with all-cause mortality in participants with chronic kidney disease. 
Table 3 HRs of all-cause mortality categorised by the fluid intake in the non-CKD and CKD group

\begin{tabular}{|c|c|c|c|c|c|c|c|c|}
\hline \multirow[b]{3}{*}{ Variables } & \multicolumn{4}{|l|}{ Non-CKD ( $\mathrm{N}=933)$} & \multicolumn{4}{|l|}{ CKD group ( $\mathrm{N}=1249)$} \\
\hline & \multicolumn{2}{|l|}{ Unadjusted model } & \multicolumn{2}{|l|}{ Totally adjusted model } & \multicolumn{2}{|l|}{ Unadjusted model } & \multicolumn{2}{|l|}{ Totally adjusted model } \\
\hline & HR (95\% Cl) & p Value & HR (95\% Cl) & p Value & HR (95\% Cl) & p Value & HR (95\% Cl) & p Value \\
\hline \multicolumn{9}{|c|}{ Total daily fluid intake (L/day) } \\
\hline$<2.147$ & 1 & Reference & 1 & Reference & 1 & Reference & 1 & Reference \\
\hline 2.147 to 2.789 & $0.881(0.695$ to 1.117$)$ & 0.295 & $1.016(0.791$ to 1.307$)$ & 0.899 & $0.944(0.754$ to 1.181$)$ & 0.613 & $0.933(0.737$ to 1.181$)$ & 0.562 \\
\hline 2.789 to 3.576 & $0.802(0.631$ to 1.019$)$ & 0.071 & $0.928(0.720$ to 1.196$)$ & 0.563 & $0.939(0.750$ to 1.177$)$ & 0.586 & $0.914(0.712$ to 1.173$)$ & 0.481 \\
\hline$\geq 3.576$ & $0.667(0.524$ to 0.850$)$ & 0.001 & $1.002(0.768$ to 1.308$)$ & 0.985 & $0.720(0.561$ to 0.923$)$ & 0.010 & 0.741 (0.566 to 0.970$)$ & 0.029 \\
\hline
\end{tabular}

Table 4 HRs of cardiovascular mortality categorised by fluid intake in the non-CKD and CKD group

\begin{tabular}{|c|c|c|c|c|c|c|c|c|}
\hline \multirow[b]{3}{*}{ Variables } & \multicolumn{4}{|l|}{ Non-CKD (N=933) } & \multicolumn{4}{|l|}{ CKD group $(\mathrm{N}=1249)$} \\
\hline & \multicolumn{2}{|l|}{ Unadjusted model } & \multicolumn{2}{|l|}{ Totally adjusted model } & \multicolumn{2}{|l|}{ Unadjusted model } & \multicolumn{2}{|l|}{ Totally adjusted model } \\
\hline & HR (95\% Cl) & p Value & HR (95\% Cl) & p Value & HR (95\% Cl) & p Value & HR (95\% Cl) & p Value \\
\hline \multicolumn{9}{|c|}{ Total daily fluid intake (L/day) } \\
\hline$<2.147$ & 1 & Reference & 1 & Reference & 1 & Reference & 1 & Reference \\
\hline 2.147 to 2.789 & $0.915(0.721$ to 1.162$)$ & 0.468 & $0.953(0.737$ to 1.233$)$ & 0.716 & $0.853(0.680$ to 1.070$)$ & 0.141 & 0.847 (0.666 to 1.079$)$ & 0.179 \\
\hline 2.789 to 3.576 & $0.819(0.642$ to 1.045$)$ & 0.108 & 0.888 (0.681 to 1.157$)$ & 0.379 & $0.844(0.672$ to 1.060$)$ & 0.145 & $0.870(0.655$ to 1.091$)$ & 0.198 \\
\hline$\geq 3.576$ & $0.878(0.688$ to 1.120$)$ & 0.296 & $0.985(0.753$ to 1.288$)$ & 0.914 & 0.841 (0.655 to 1.079$)$ & 0.173 & $0.827(0.643$ to 1.115$)$ & 0.236 \\
\hline
\end{tabular}

Adjusted for age, gender, race-ethnicity, BMl, waist, white cell count, eGFR, HDL cholesterol, apolipoprotein Al, glucose, plasma fibrinogen, albumin, C reactive protein, smoke, cancer, emphysema, DM and arthritis.

BMI, body mass index; CKD, chronic kidney disease; DM, diabetes mellitus; eGFR, estimated glomerular filtration rate; HDL, high-density lipoprotein.

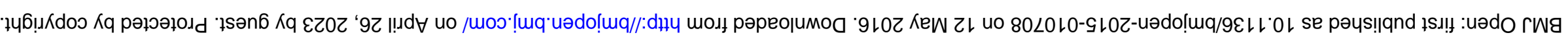


with water but not with total fluid intake and provided an explanation for the discordance.

To date, there has been no clear evidence that healthy individuals benefit from drinking water abundantly, but also none conceding a lack of benefit from doing so. In population-based cohorts, the risk of CKD and the annual decline in eGFR had been shown to be inversely correlated with $24 \mathrm{~h}$ fluid intake and urine volume. ${ }^{4}$ In healthy volunteers, increased fluid intake improved the ability of the kidneys to excrete sodium, whereas the antidiuretic action of vasopressin resulted in substantial sodium retention. ${ }^{18}$ Patients with higher urine volumes showed higher BPs, lower serum sodium levels and frankly hypotonic urine, suggesting a 'pushing diuresis'. Nevertheless, the underlying mechanisms of the responses of normal and injured kidneys to chronic changes in water intake are still poorly understood, and the clinical effect of daily fluid consumption on mortality and kidney function has seldom been addressed. Using a non-institutionalised, geographically dispersed and ethnically diverse national population-based sample, our study demonstrated that individuals in the highest quartile of daily fluid intake showed significantly better overall survival than participans in the lowest quartile of daily fluid intake with respect to all-cause mortality regardless of controlling for demographic factors and comorbidities at baseline. However, higher daily fluid intake was not associated with a reduction in long-term cardiovascular mortality. Baseline cross-sectional analysis revealed that there was no evidence for the association between lower daily fluid intake, and the risk of CKD and CVD.

The findings in the present study were not in line with those of the prior studies concerning all-cause mortality. ${ }^{19}{ }^{20}$ The discrepancies were likely because they used only estimated total fluid content of food and beverages but not water. In addition, body mass index (BMI) in patients with CKD showed a relatively consistent U-shaped association with all-cause mortality, with the best outcomes observed in overweight $\left(25-30 \mathrm{~kg} / \mathrm{m}^{2}\right)$ and mildly obese $\left(30-35 \mathrm{~kg} / \mathrm{m}^{2}\right)$ patients. ${ }^{21}$ BMI levels above or below the nadirs $\left(30-35 \mathrm{~kg} / \mathrm{m}^{2}\right)$ indicated associations with increased mortality independent of the severity of CKD. In our study, the mean BMI (27.82 kg/ $\mathrm{m}^{2}$ ) of individuals in the highest quartiles of fluid intake was closer to the nadirs $\left(30-35 \mathrm{~kg} / \mathrm{m}^{2}\right)$ rather than participants in the lowest quartiles of fluid intake, resulting in better survival.

Emerging evidence has shown the negative effect of low habitual fluid intake on some chronic diseases, such as asthma, cardiovascular disease, diabetic hyperglycaemia and some cancers. ${ }^{22}$ Chronic fluid deficit may be a precipitating factor of mortality in a vulnerable population. A cross-sectional study, performed by Rasouli et $a l^{23}$ noted a positive link between serum osmolality and the prevalence of coronary artery disease, leading to a hypothesis that dehydration may play an important role in atherosclerosis. In our study, there was no benefit with respect to cardiovascular mortality in patients with CKD with higher daily fluid intake, which was consistent with the Netherlands study reported by Leurs et al. In that cohort study of 3970 individuals aged between 55 and 69 years, total fluid intake was, in neither men nor women, associated with ischaemic heart disease or stroke mortality over a 10-year follow-up period. ${ }^{24}$ Inconsistent with the findings obtained in our study, a longitudinal study reported by Chan et al, ${ }^{10}$ of 20297 individuals living in California, revealed that high daily intake of water (five or more glasses) compared with low daily intake (two or fewer glasses), was associated with a relative risk of cardiovascular mortality of 0.46 in men and 0.59 in women. The possible explanation for the inconsistency of findings is that the study by Chan $e t a l^{10}$ has much greater power than our study, with vastly greater numbers of participants and, also, they looked specifically at water and not simply at total fluid intake, so it is more in keeping with the positive findings of Sontrop et al. ${ }^{17}$ Since different measures of fluid intake and analytical mode may be confounded and overshadowed by baseline risks of CVD, different conclusions were made in these studies. It is an important weakness of the comparisons between the observational studies. Another potential explanation for the difference in cardiovascular mortality may be the high prevalence $(97 \%)$ of early CKD (stage $1-3$ ) in the CKD group in our study, attributing to lower burden of cardiovascular disease and associated mortality.

Although the existence of a link between increased fluid intake and reduced renal function in patients with CKD was plausible, there was little empirical evidence to establish a direct relationship between the two parameters. In a cross-sectional study from NHANES, higher water intake was associated with a non-significant adjusted risk of CKD compared with lowest water intake. ${ }^{19}$ However, clinical evidence regarding the beneficial role of fluid intake on the improvement of renal function in patients with CKD remains controversial. Although the mechanisms driving these changes have yet to be clarified, it is tempting to speculate that the suppression of arginine vasopressin (AVP) induced by fluid ingestion impairs the worsening severity of renal damage and albuminuria by modulating tubular cell growth and increasing renal plasma flow along with glomerular hyperfiltration. ${ }^{25} 26$

There were several limitations to the present study. Although the Dietary Food Frequency Questionnaire used in the NHANES III survey was validated, $24 \mathrm{~h}$ dietary recall may not provide an indication of long-term diet nor an accurate representation of actual intake. Moreover, the interpretation of our observations was limited by a failure to identify the composition of ingested fluids, reflecting the pivotal role of water and salt intake on volume status and plasma osmolarity. It would be more precise to obtain additional data during the follow-up period. The interaction between daily fluid intake and changes in renal function over time was not analysed, because fluid intake and other clinical 
variables were measured only once at enrolment of the cross-sectional data of NHANES III. Regarding its presence, CVD was determined via self-reporting in the NHANES III survey, thereby leading to the recall bias or misclassification.

In summary, participants with fluid intake $\geq 3.576 \mathrm{~L} /$ day had more favourable all-cause mortality than participants with fluid intake $\leqq 2.147 \mathrm{~L} /$ day, but not more favourable cardiovascular mortality. Although no prospective randomised controlled trial was available, it appears that adequate hydration provides some advantage in patients with CKD. Providing specific recommendations regarding fluid intake in patients with CKD was not appropriate before determining if fluid or water intake was both beneficial and safe. Understanding the pathophysiological mechanisms of fluid intake may improve disease progression and outcomes in patients with CKD, and warrants further investigation.

\section{Author affiliations}

${ }^{1}$ Division of Family Medicine, Tri-Service General Hospital; and School of Medicine, National Defense Medical Center, Taipei, Taiwan (ROC)

${ }^{2}$ Division of Geriatric Medicine, Department of Family and Community Medicine, Tri-Service General Hospital; and School of Medicine, National Defense Medical Center, Taipei, Taiwan (ROC)

${ }^{3}$ Graduate Institute of Medical Sciences, National Defense Medical Center, Taipei, Taiwan (ROC)

${ }^{4}$ Division of Nephrology, Department of Medicine, Taoyuan General Hospital, Taoyuan City, Taiwan (ROC)

${ }^{5}$ Institute of Clinical Medicine, National Yang-Ming University, Taipei, Taiwan (ROC)

Contributors L-WW and W-LC contributed to the design of the study, were responsible for the management and retrieval of data, contributed to initial data analysis and interpretation, and drafted the initial manuscript. L-WW, W-LC, F-YL, Y-SS, H-FY, C-CW, C-ML and Y-TT designed the intervention and decided on the data collection methods. L-WW and W-LC were also responsible for the data analysis decisions. Y-TT conceptualised and designed the study, supervised all aspects of the study, critically reviewed and revised the manuscript, and approved the final manuscript as submitted.

Funding This research received no specific grant from any funding agency in the public, commercial or not-for-profit sectors.

Competing interests None declared.

Provenance and peer review Not commissioned; externally peer reviewed.

Data sharing statement All data are available from the website of NHANES (questionnaires, data sets and related documentation). http://www.cdc.gov/ nchs/nhanes/nhanes_questionnaires.htm.

Open Access This is an Open Access article distributed in accordance with the Creative Commons Attribution Non Commercial (CC BY-NC 4.0) license, which permits others to distribute, remix, adapt, build upon this work noncommercially, and license their derivative works on different terms, provided the original work is properly cited and the use is non-commercial. See: http:// creativecommons.org/licenses/by-nc/4.0/

\section{REFERENCES}

1. Astor BC, Matsushita K, Gansevoort RT, et al. Lower estimated glomerular filtration rate and higher albuminuria are associated with mortality and end-stage renal disease. A collaborative meta-analysis of kidney disease population cohorts. Kidney Int 2011;79:1331-40.

2. Gansevoort RT, Matsushita K, van der Velde M, et al. Chronic Kidney Disease Prognosis Consortium: lower estimated GFR and higher albuminuria are associated with adverse kidney outcomes. A collaborative meta-analysis of general and high-risk population cohorts. Kidney Int 2011;80:93-104.

3. European Food Safety Authority (EFSA) Panel on Dietetic Products, Nutrition, and Allergies. Scientific opinion on dietary reference values for water. EFSA J 2010;8:1459.

4. Clark WF, Sontrop JM, Macnab JJ, et al. Urine volume and change in estimated GFR in a community-based cohort study. Clin J Am Soc Nephrol 2011;6:2634-41.

5. Strippoli GF, Craig JC, Rochtchina E, et al. Fluid and nutrient intake and risk of chronic kidney disease. Nephrology (Carlton) 2011;16:326-34.

6. Bankir L, Bouby N, Trinh-Trang-Tan MM. Vasopressin-dependent kidney hypertrophy: role of urinary concentration in protein-induced hypertrophy and in the progression of chronic renal failure. $A m \mathrm{~J}$ Kidney Dis 1991;17:661-5.

7. Bouby N, Bachmann S, Bichet D, et al. Effect of water intake on the progression of chronic renal failure in the 5/6 nephrectomized rat. Am J Physiol Renal Physiol 1990;258:F973.

8. Sugiura $\mathrm{T}$, Yamauchi $\mathrm{A}$, Kitamura $\mathrm{H}$, et al. High water intake ameliorates tubulointerstitial injury in rats with subtotal nephrectomy: possible role of TGF-beta. Kidney Int 1999;55:1800-10.

9. Nagao S, Nishii K, Katsuyama M, et al. Increased water intake decreases progression of polycystic kidney disease in the PCK Rat. $J$ Am Soc Nephrol 2006;17:2220-7.

10. Chan J, Knutsen SF, Blix GG, et al. Water, other fluids, and fatal coronary heart disease: the Adventist Health Study. Am J Epidemiol 2002;155:827-33.

11. Hu FB, Rimm E, Smith-Warner SA, et al. Reproducibility and validity of dietary patterns assessed with a food-frequency questionnaire. Am J Clin Nutr 1999;69:243-9.

12. Feskanich D, Rimm EB, Giovannucci EL, et al. Reproducibility and validity of food intake measurements from a semiquantitative food frequency questionnaire. J Am Diet Assoc 1993;93:

790-6.

13. Centers for Disease Control and Prevention, National Center for Health Statistics. Third National Health and Nutrition Examination Survey data. Hyattsville, MD: US Department of Health and Human Services, Centers for disease Control and Prevention, 1988-1994.

14. Center for Disease Control and Prevention (CDC). Third National Health and Nutrition Examination Survey (NHANES III), 1988-1994, linked mortality file: matching methodology. Hyattsville, MD: Centers for Disease Control and Prevention, 2005.

15. Kao TW, Chang YW, Chou CC, et al. White blood cell count and psychomotor cognitive performance in the elderly. Eur J Clin Invest 2011;41:513-20.

16. National Center for Health Statistics. The Third National Health and Nutrition Examination Survey (NHANES III 1988-94) Reference Manuals and Reports. Bethesda, MD: Centers for Disease Control and Prevention Press, 1996.

17. Sontrop JM, Dixon SN, Garg AX, et al. Association between water intake, chronic kidney disease, and cardiovascular disease: a cross-sectional analysis of NHANES data. Am J Nephrol 2013;37:434-42.

18. Bankir L, Bichet DG, Bouby N. Vasopressin V2 receptors, ENaC and sodium reabsorption: a risk factor for hypertension? Am J Physiol Renal Physiol 2010;299:F917-28.

19. Palmer SC, Wong G, Iff S, et al. Fluid intake and all-cause mortality, cardiovascular mortality, and kidney function: a population-based longitudinal cohort study. Nephrol Dial Transplant 2014;29:1377-84.

20. Ritchie J, O'Donoghue D. Fluid intake and mortality: drinking in the data. Nephrol Dial Transplant 2014;29:1265-7.

21. Lu JL, Kalantar-Zadeh K, Ma JZ, et al. Association of body mass index with outcomes in patients with CKD. J Am Soc Nephrol 2014;25:2088-96.

22. Manz F, Wentz A. The importance of good hydration for the prevention of chronic diseases. Nutr Rev 2005;63:S2-5.

23. Rasouli M, Kiasari AM, Arab S. Indicators of dehydration and haemoconcentration are associated with the prevalence and severity of coronary artery disease. Clin Exp Pharmacol Physio 2008;35:889-94.

24. Leurs LJ, Schouten LJ, Goldbohm RA, et al. Total fluid and specific beverage intake and mortality due to IHD and stroke in the Netherlands Cohort Study. Br J Nutr 2010;104:1212-21.

25. Torres VE. Vasopressin in chronic kidney disease: an elephant in the room? Kidney Int 2009;76:925-8.

26. Bolignano D, Zoccali C. Vasopressin beyond water: implications for renal diseases. Curr Opin Nephrol Hypertens 2010;19:499-504. 